\title{
A Theoretical Model of pH-Based Potentiometric Biosensor Based on Immobilized Enzyme Membrane
}

\author{
Jeganathan Saranya1, Lakshmanan Rajendran ${ }^{2 *}$, Mariappan Uma Maheswari ${ }^{3}$ \\ ${ }^{1}$ Department of Mathematics, Mother Teresa Women's University, Kodaikanal, India \\ ${ }^{2}$ Department of Mathematics, Sethu Institute of Technology, Kariapatti, India \\ ${ }^{3}$ Department of Mathematics, Kamaraj College of Engineering and Technology, Virudhunagar, India \\ Email: saranyajmaths2008@gmail.com, raj_sms@rediffmail.com,umathiru84@gmail.com
}

Received 4 March 2016; accepted 19 April 2016; published 22 April 2016

Copyright (C) 2016 by authors and Scientific Research Publishing Inc.

This work is licensed under the Creative Commons Attribution International License (CC BY). http://creativecommons.org/licenses/by/4.0/

(c) (i) Open Access

\begin{abstract}
A theoretical model for the non steady-state response of a pH-based potentiometric biosensor immobilizing organophosphorus hydrolase (OPH) is discussed. The model is based on a system of five coupled nonlinear reaction-diffusion equations under non steady-state conditions for enzyme reactions occurring in potentiometric biosensor that describes the concentration of substrate and hydrolysis products within the membrane. New approximate analytical expressions for the concentration of the substrate (organophosphorus pesticides (OPs)) and products are derived for all values of Thiele modulus and buffer concentration using new approach of homotopy perturbation method. The analytical results are also compared with numerical ones and a good agreement is obtained. The obtained results are valid for the whole solution domain.
\end{abstract}

\section{Keywords}

Mathematical Modeling, Reaction-Diffusion, pH-Based Potentiometric Biosensor, Asymptotic Methods, New Homotopy Perturbation Method

\section{Introduction}

A potentiometric biosensor is a type of chemical sensor that may be used to find the concentration of some components of the analyte. These sensors measure the electrical potential of an electrode when no voltage is present. The potentiometric biosensors have been widely used in environmental, medical and industrial applica-

${ }^{*}$ Corresponding author.

How to cite this paper: Saranya, J., Rajendran, L. and Maheswari, M.U. (2016) A Theoretical Model of pH-Based Potentiometric Biosensor Based on Immobilized Enzyme Membrane. American Journal of Analytical Chemistry, 7, 363-377. 
tions [1]. Also potentiometric biosensor can be used for detection of all OPs but they don't have low enough limits of detection [2].

The theoretical modeling of biosensors involves solving the system of linear/non-linear reaction-diffusion equations for substrate and product with a term containing a rate of biocatalytical transformation of substrate. The complications of modeling arise due to solving the partially differential equations with non-linear reaction term and with complex initial and boundary conditions. The modeling of biosensor is analyzed by numerical [1] and analytical method [3] of partial differential equation with various boundary conditions. Recently Meena and Rajendran (2010) discussed a theoretical model of a pH-based potentiometric biosensor immobilizing organophosphorus hydrolase (OPH) for steady state conditions [4].

Rahamathunissa and Rajendran (2008) implemented He's variational iteration method in nonlinear boundaryvalue problems in enzyme substrate reaction diffusion processes in amperometric biosensor [5]. Manimozhi et al. [6] presented the solution of steady-state substrate concentration in the action of biosensor response with mixed enzyme kinetics under a Michalis-Menten scheme. Analytical solutions for the steady-state current at a microdisk chemical sensor have been reported by Dong and Che [7] and by Lyons et al. [7] [8]. Recently, Eswari and Rajendran [9] derived the concentration profile of the product of the enzyme reaction and the electrode current for all values of Michalis-Menten constant using the Homotopy perturbation method.

To our knowledge, no general analytical expressions of the concentrations of the substrate, hydrolysis products, added external buffer and hydrogen ions have been reported for all values of parameters. The purpose of this communication is to derive an analytical expression of non-steady state concentrations of OPs and the deprotonation products for all values of reaction parameter using new homotopy perturbation method.

\section{Mathematical Formulation of the Problem}

The complete description of the problem is given in [4] [10]. For the sake of completeness the brief description is given in this section and Appendix-A. A schematic diagram of the $\mathrm{pH}$-based potentiometric biosensor immobilizing organophosphorus hydrolase (OPH) is represented in Figure 1.

In this figure, $S$ denotes the substrate of organophosphorus pesticides (OPs). $P_{h} H$ and $Z H$ are represent the hydrolysis products of organophosphodiester and alcohol respectively. $A H$ is the added external buffer and $P_{h}^{-}, Z^{-}, A^{-}, H^{+}$are the deprotonation products. The general scheme that represents an enzyme-cata- lyzed reaction within enzyme membrane can be written as follows:

$$
\begin{gathered}
S \underset{\mathrm{H}_{2} \mathrm{O}}{\stackrel{E}{\longrightarrow}} P_{h} H+Z H \\
P_{h} H \underset{k_{p}^{\prime}}{\stackrel{k_{p}}{\Leftrightarrow}} P_{h}^{-}+H^{-}
\end{gathered}
$$

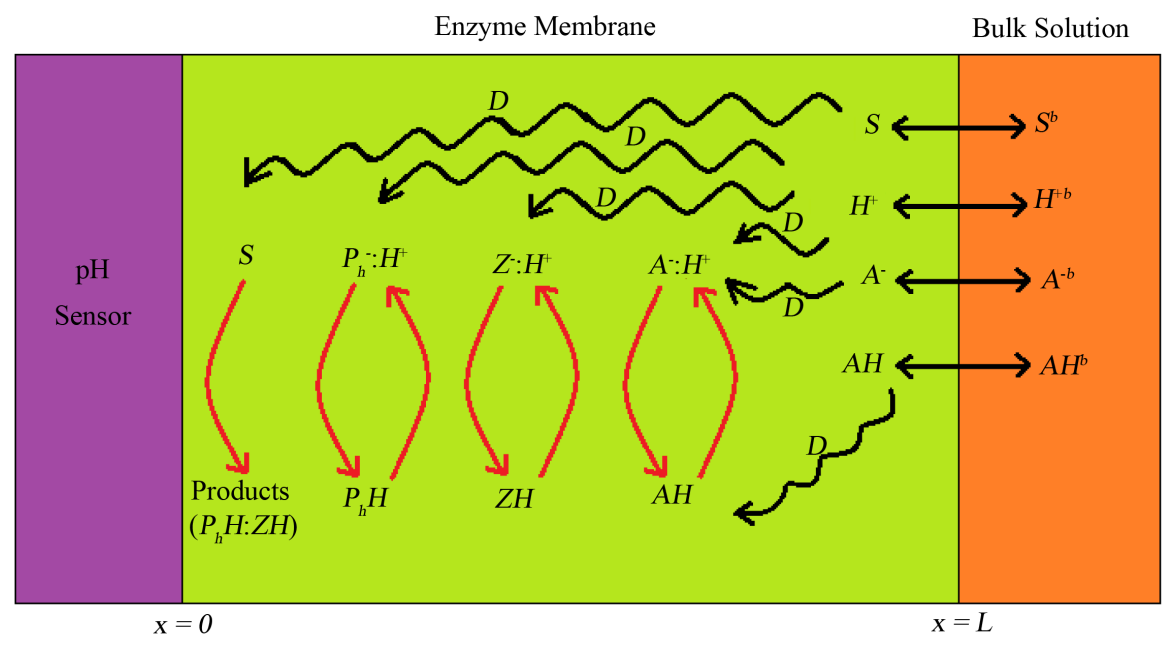

Figure 1. Schematic representation of pH-based potentiometric biosensor immobilizing OPs (organophosphorus pesticides). 


$$
\begin{aligned}
Z H \underset{k_{Z}^{\prime}}{\stackrel{k_{Z}}{\Leftrightarrow}} Z^{-}+H^{+} \\
A H \underset{k_{A}^{\prime}}{\stackrel{k_{A}}{\Leftrightarrow}} A^{-}+H^{+} .
\end{aligned}
$$

The non-linear reaction-diffusion equations for non-steady state condition can be described as follows

$$
\frac{\partial C_{i}}{\partial t}=D_{i} \frac{\partial^{2} C_{i}}{\partial x^{2}}+R\left(C_{i}\right)
$$

where $C_{i}$ is the concentration of species, $D_{i}$ is the diffusion coefficient and $R\left(C_{i}\right)$ is the reaction rate. The reaction rate is a non-linear function of concentration of substrate. The reaction rate is non-linear with respect to substrate because of product inhibition, saturation of the enzyme with substrate, reverse reaction and enzyme

\begin{tabular}{|c|c|c|}
\hline Symbol & Usual units & Definition \\
\hline$[S]$ & $\mathrm{mol} / \mathrm{cm}^{3}$ & Concentration of substrate \\
\hline$\left[P_{h} H\right]$ & $\mathrm{mol} / \mathrm{cm}^{3}$ & Hydrolysis products of organophosphodiester \\
\hline$[Z H]$ & $\mathrm{mol} / \mathrm{cm}^{3}$ & Concentration of hydrolysis products of alcohol \\
\hline$[A H]$ & $\mathrm{mol} / \mathrm{cm}^{3}$ & Added external buffer \\
\hline$P_{h}^{-}, Z^{-}, A^{-}, H^{+}$ & $\mathrm{mol} / \mathrm{cm}^{3}$ & Deprotonation products \\
\hline$C_{i}$ & $\mathrm{~mol} / \mathrm{cm}^{3}$ & Concentration of the species \\
\hline$D_{i}$ & $\mathrm{~cm}^{2} / \mathrm{s}$ & Diffusion coefficients \\
\hline$R, r_{P_{h} H}, r_{Z H}, r_{A H}, r_{A H}$ & $\mu \mathrm{mol} / \mathrm{min}$ & Rate of reactions \\
\hline$k_{1}, k_{-1}$ & $\mathrm{~cm} / \mathrm{s}$ & Rate constant for the formation of the Michaelis complex \\
\hline$k_{2}$ & $\mathrm{~cm} / \mathrm{s}$ & Rate constant for the chemical transformation \\
\hline$k_{3}$ & $\mathrm{~cm} / \mathrm{s}$ & Rate constant for product dissociation \\
\hline$[E]_{t}$ & $\mathrm{~mol} / \mathrm{cm}^{3}$ & Enzyme concentration \\
\hline$\overline{[S]}$ & None & Dimensionless concentration of $S$ \\
\hline$\overline{\left[P_{h}\right]_{T}}$ & None & Dimensionless concentration of $P_{h} H$ \\
\hline$\overline{[Z]_{T}}$ & None & Dimensionless concentration of $\mathrm{ZH}$ \\
\hline$\overline{[A]_{T}}$ & None & Dimensionless concentration of $A H$ \\
\hline$H^{+}$ & None & Dimensionless concentration of hydrogen ions \\
\hline$x$ & $\mathrm{~cm}$ & Distance \\
\hline$L$ & $\mathrm{~cm}$ & Thickness of the enzyme membrane \\
\hline $\bar{x}$ & None & Dimensionless distance \\
\hline$a$ & None & Thiele modulus \\
\hline$K_{m}$ & $\mathrm{~mol} / \mathrm{cm}^{3}$ & Michaelis-Menten constant. \\
\hline$K_{P_{h}} / K_{m}, \quad K_{Z} / K_{m}, K_{A} / K_{m}$ & None & Dimensionless equilibrium constants \\
\hline$\tau=D t / L^{2}$ & None & Dimensionless time \\
\hline$\alpha=a^{2} /\left(\overline{[S]}^{b}+1\right)$ & None & Dimensionless parameter \\
\hline$f_{n}=\left(\pi^{2}(2 n+1)^{2}+4 \alpha\right) / 4$ & None & Dimensionless parameter \\
\hline
\end{tabular}
loading. The nomenclature is also presented in Table 1.

\section{Table 1. Nomenclature.}




\section{Dimensionless Form}

The dimensionless reaction-diffusion equations for non-steady state condition can be written as follows (Appen$\operatorname{dix}$ A):

$$
\begin{gathered}
\frac{\partial \overline{[S]}}{\partial \tau}=\frac{\partial^{2} \overline{[S]}}{\partial \bar{x}^{2}}-a^{2} \frac{\overline{[S]}}{\overline{[S]}+1} \\
\frac{\partial \overline{\left[P_{h}\right]_{T}}}{\partial \tau}=\frac{\partial^{2} \overline{\left[P_{h}\right]_{T}}}{\partial \bar{x}^{2}}+a^{2} \frac{\overline{[S]}}{\overline{[S]}+1} \\
\frac{\partial \overline{[Z]_{T}}}{\partial \tau}=\frac{\partial^{2} \overline{[Z]_{T}}}{\partial \bar{x}^{2}}+a^{2} \frac{\overline{[S]}}{\overline{[S]}+1} \\
\frac{\partial \overline{[A]_{T}}}{\partial \tau}=\frac{\partial^{2} \overline{[A]_{T}}}{\partial \bar{x}^{2}} \\
\frac{\partial\left[H^{+}\right]_{T}}{\partial \tau}=\frac{\partial^{2} \overline{\left[H^{+}\right]_{T}}}{\partial \bar{x}^{2}}+2 a^{2} \frac{\overline{[S]}}{\overline{[S]}+1}
\end{gathered}
$$

where $\left[P_{h}\right]_{T},[Z]_{T}$ and $[A]_{T}$ denotes the sum of dissociated and undissociated concentrations of the species $P_{h} H, Z H$, and $A H$ respectively and $\left[H^{+}\right]_{T}$ is the concentration of hydrogen ions. Here the Thiele modulus $a$, which represents the ratio of the characteristic time of the enzymatic reaction to that of the substrate diffusion is

$$
a^{2}=\frac{L^{2} V_{\max }}{D k_{m}} .
$$

The initial and boundary conditions for the above equations becomes

$$
\begin{gathered}
\overline{C_{i}}(\bar{x}, 0)=0 \text {; for } \overline{C_{i}}=\overline{[S]}, \overline{\left[P_{h}\right]_{T}}, \overline{[Z]_{T}}, \overline{[A]_{T}}, \overline{\left[H^{+}\right]_{T}} \\
\frac{\mathrm{d} \overline{C_{i}}}{\mathrm{~d} \bar{x}}(0)=0 ; \text { for } \overline{C_{i}}=\overline{[S]}, \overline{\left[P_{h}\right]_{T}}, \overline{[Z]_{T}}, \overline{[A]_{T}}, \overline{\left[H^{+}\right]_{T}} \\
\overline{C_{i}}(1)=\overline{C_{i}^{b}} ; \text { for } \overline{C_{i}}=\overline{[S]}, \overline{[A]_{T}}, \overline{\left[H^{+}\right]_{T}} ; \overline{\left[P_{h}\right]_{T}}(1)=\overline{[Z]_{T}}(1)=0 .
\end{gathered}
$$

A graphical representation of the boundary conditions of this system conditions can be seen in Figure 2.

\section{Analytical Expression of Concentration of Substrate and Products Using New Homotopy Perturbation Method (New HPM) and Laplace Transform Technique}

With the rapid development of nonlinear science, there appears an ever-increasing interest of scientists and engineers in the approximate analytical asymptotic techniques for nonlinear problems [11]. It is very difficult to solve nonlinear problems either numerically or theoretically. Perturbation methods provide the most versatile tools available in nonlinear analysis of engineering problems, and they are constantly being developed and applied to ever more complex problems. Homotopy perturbation method was first proposed by the He [12]. Recently, a new approach to HPM is presented to solve the nonlinear problem and this gives a simple approximate solution in the zeroth iteration [13]. By using this new homotopy perturbation method and Laplace transform technique (Appendix B), the concentrations of substrate and products can be obtained as follows:

$$
\overline{[S]}(\bar{x}, \tau)=\overline{[S]^{b}}\left\{\frac{\cosh \sqrt{\alpha} \bar{x}}{\cosh \sqrt{\alpha}}-\sum_{n=0}^{\infty}\left[\frac{(-1)^{n} \pi(2 n+1) \mathrm{e}^{-f_{n} \tau} \cos ((2 n+1) \pi \bar{x} / 2)}{f_{n}}\right]\right\}
$$




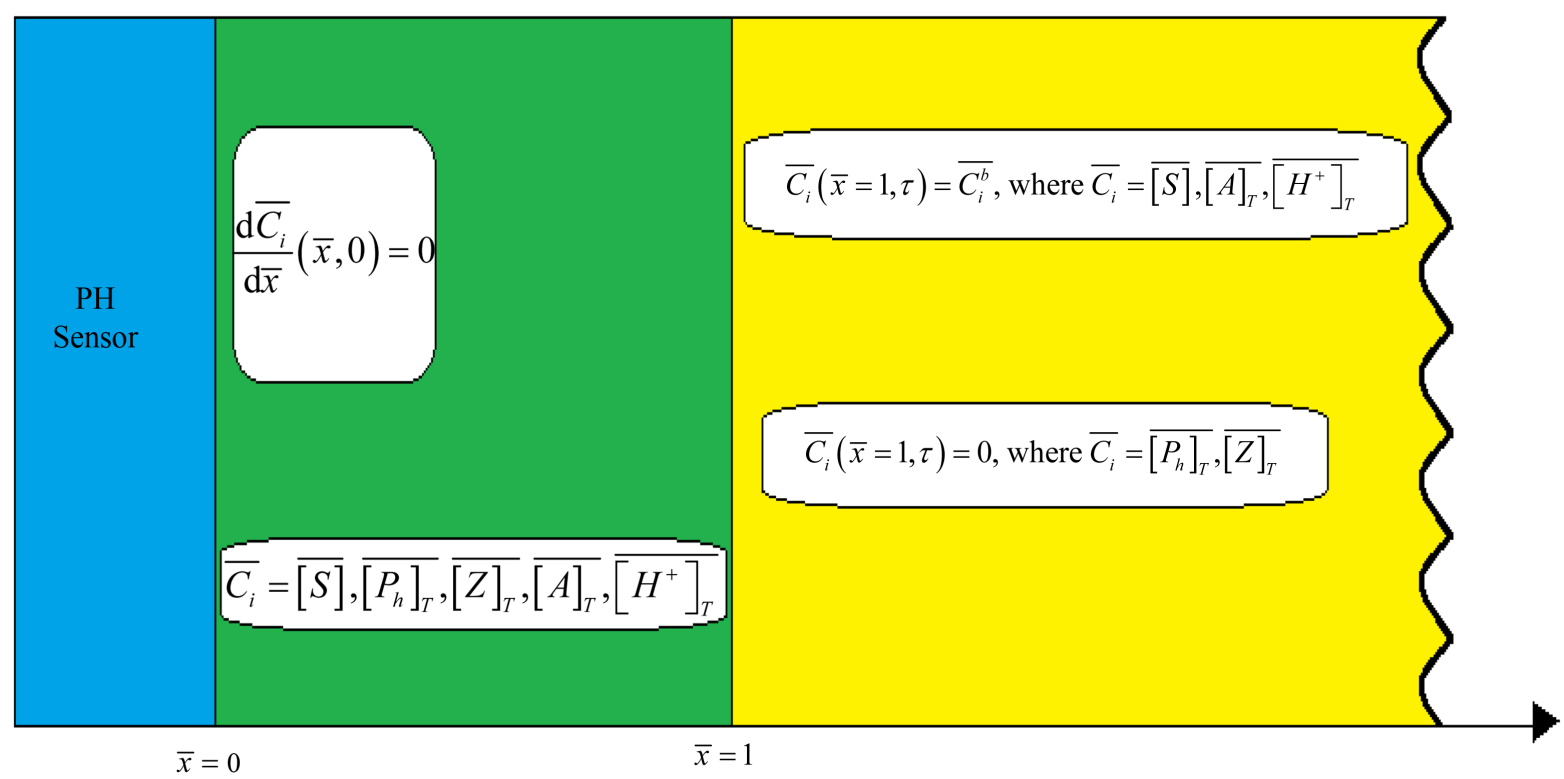

Figure 2. Boundary conditions employed in the pH-based potentiometric biosensor for the substrate $\overline{[S]}$, products $\overline{\left[P_{h}\right]_{T}}, \overline{[Z]_{T}}, \overline{\left[H^{+}\right]_{T}}$ and added external buffer concentration of species $(A H) \overline{[A]_{T}}$.

$$
\begin{aligned}
& \overline{[Z]_{T}}(\bar{x}, \tau)=\overline{[S}^{b}\left\{1+\frac{4}{\pi} \sum_{n=1}^{\infty}\left[\frac{(-1)^{n} \mathrm{e}^{-\left((2 n-1)^{2} \pi^{2} \tau / 4\right)} \cos ((2 n-1) \pi \bar{x} / 2)}{(2 n-1)}\right]\right\}-\overline{[S]}(\bar{x}, \tau) \\
& \overline{\left[H^{+}\right]_{T}}(\bar{x}, \tau)=\left(2[\bar{S}]^{b}+{\overline{\left[H^{+}\right]_{T}}}_{T}^{b}\right)\left\{1+\frac{4}{\pi} \sum_{n=1}^{\infty}\left[\frac{(-1)^{n} \mathrm{e}^{-\left((2 n-1)^{2} \pi^{2} \tau / 4\right)} \cos ((2 n-1) \pi \bar{x} / 2)}{(2 n-1)}\right]\right\}-2[\bar{S}](\bar{x}, \tau) \\
& \overline{\left[P_{h}\right]_{T}}(\bar{x}, \tau)=\overline{[S]^{b}}\left\{1+\frac{4}{\pi} \sum_{n=1}^{\infty}\left[\frac{(-1)^{n} \mathrm{e}^{-\left((2 n-1)^{2} \pi^{2} \tau / 4\right)} \cos ((2 n-1) \pi \bar{x} / 2)}{(2 n-1)}\right]\right\}-\overline{[S]}(\bar{x}, \tau) \\
& \overline{[A]_{T}}(\bar{x}, \tau)={\overline{[A]_{T}}}^{b}\left\{1+\frac{4}{\pi} \sum_{n=1}^{\infty}\left[\frac{(-1)^{n} \mathrm{e}^{-\left((2 n-1)^{2} \pi^{2} \tau / 4\right)} \cos ((2 n-1) \pi \bar{x} / 2)}{(2 n-1)}\right]\right\}
\end{aligned}
$$

where

$$
f_{n}=\frac{\pi^{2}(2 n+1)^{2}+4 \alpha}{4}, \alpha=\frac{a^{2}}{\overline{[S]^{b}+1} .}
$$

\section{Results and Discussion}

Equations (15) to (19) represents the general new closed-form of analytical expression for the concentrations of substrate $\overline{[S]}$, hydrolysis products $\overline{[Z]_{T}}$ and $\left[\overline{\left.P_{h}\right]_{T}}\right.$, added external buffer concentration of species $(A H)$ $\overline{[A]_{T}}$ and hydrogen ions $\left[\mathrm{H}^{+}\right]_{T}$ for non-steady state condition for all values of parameters (Thiele modulus, 
initial concentration of substrate and products). It is of interest to compare the influence of each parameter on the concentration of species for various values of the parameters.

The kinetic response of a pH-based potentiometric biosensor depends on the concentration of substrate. However, substrate concentration depends on two factors, $\alpha$ and $[S]^{b}$. The dimensionless parameter $\alpha$ depends upon $a$ and $\overline{[S]}$. " $a$ " is the Thiele modulus, which represents the ratio of the characteristic time of the enzymatic reaction to that of substrate diffusion. When the Thiele modulus " $a$ " is small, the kinetics dominates and the uptakes of the substrate are kinetically controlled. The response is under diffusion control, when the Theile modulus is large $(a>1)$, which is observed at high catalytic activity and great membrane thickness or at low Michaelis constant or diffusion coefficient values.

1) Influence of time on the concentration of species. Figures 3(a)-(f) represent concentration of the substrate

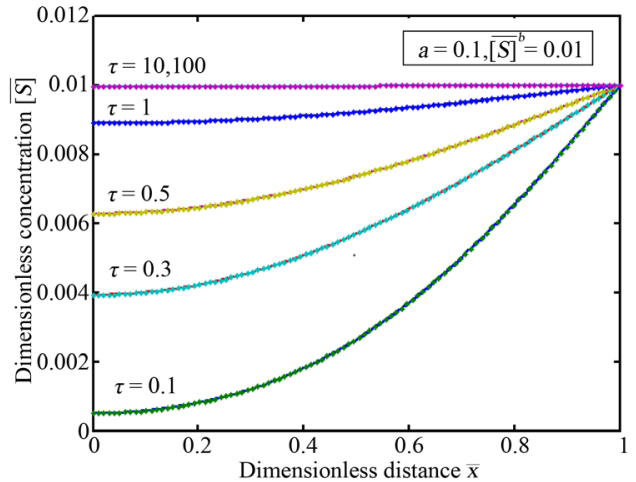

(a)

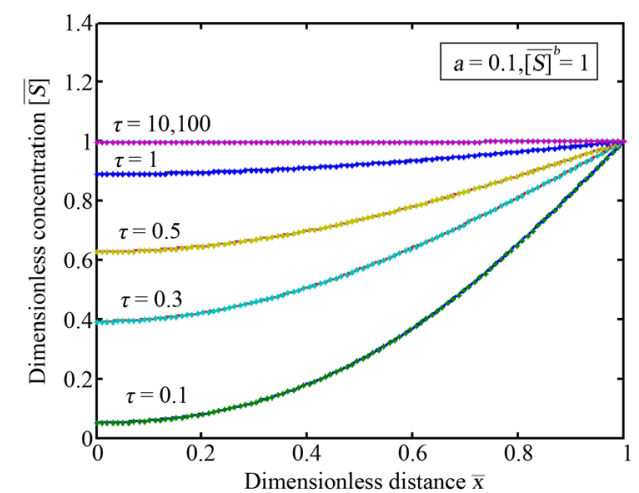

(c)

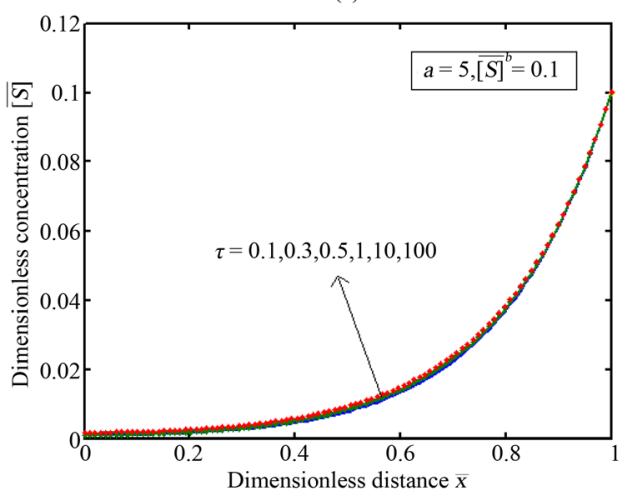

(e)

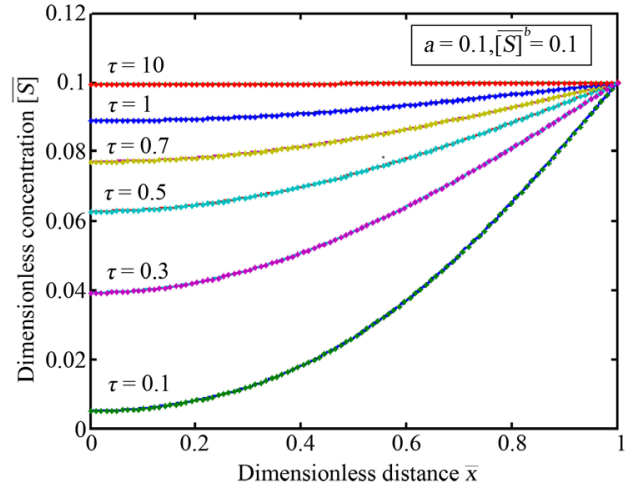

(b)

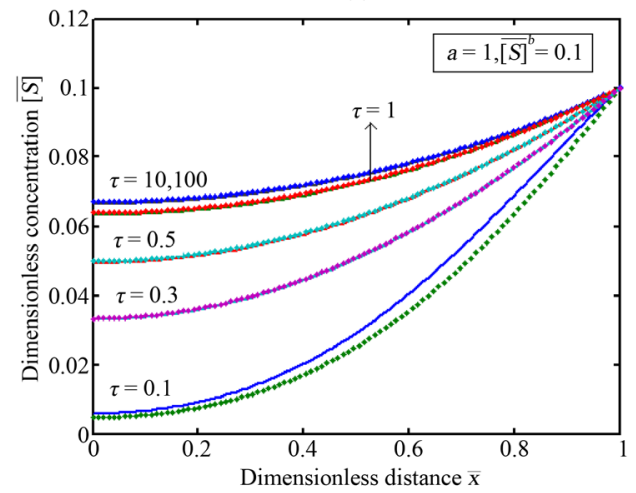

(d)

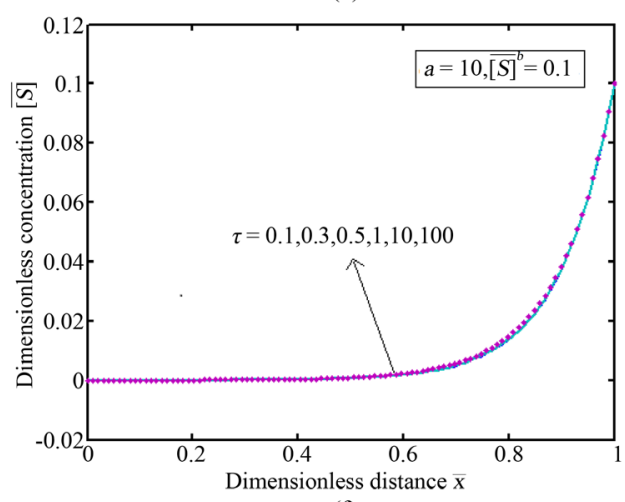

(f)

Figure 3. (a)-(f) Plot of dimensionless non-steady state concentration profiles of the substrate $\overline{[S]}$ versus dimensionless distance $\bar{x}$ for fixed values of $a$ and $\overline{[S}^{b}$ and various values of time $\tau$. Solid lines represent the Equation (15) and the dotted lines represent the numerical simulation. 
versus dimensionless distance for fixed values of $a$ and $\overline{[S}^{b}$ and various values of time $\tau$. From Figures 3(a)-(d), it is inferred that concentration of substrate increases when time $\tau$ increases. The concentration of substrate is in uniform or in steady state when, time $\tau \geq 10$ and Thiele modulus and buffer concentration are small. From Figure 3(e) and Figure 3(f), it is observed that concentration of substrate is in uniform or in steady state when, time $\tau \geq 0.1$ and Thiele modulus is large.

2) Influence of Thiele modulus on the concentration of species. The influence of Thiele modulus on the concentration of the substrate for some values of other parameters is shown in Figures 4(a)-(f). The concentration of the substrate strongly depends on Thiele modulus $a$. From this figure, it is observed that the concentration of substrate decreases when Thiele modulus increases. The concentration of substrate is in uniform or in steady state when Thiele modulus $a \leq 0.1$.

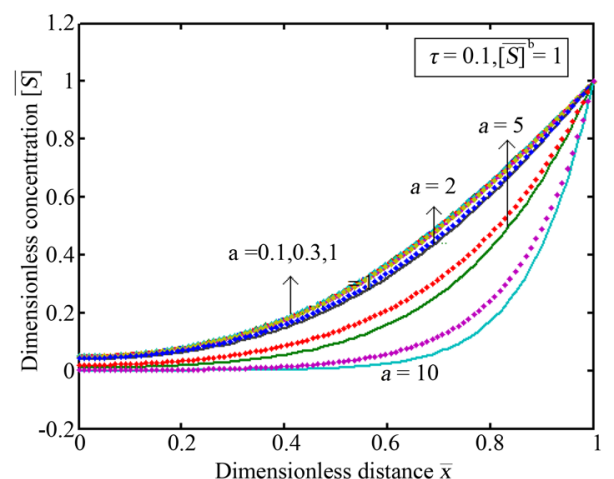

(a)

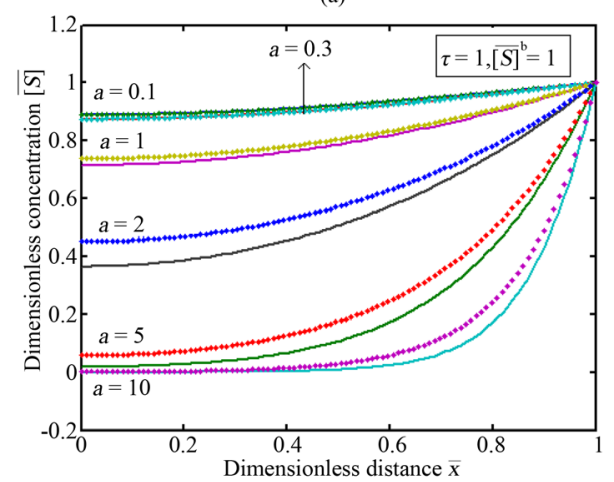

(c)

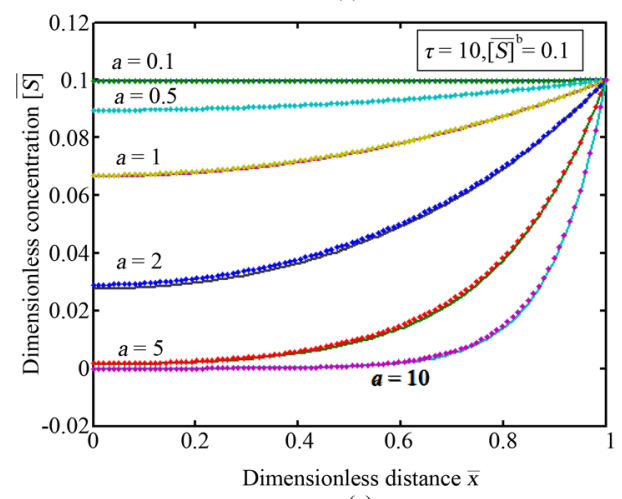

(e)

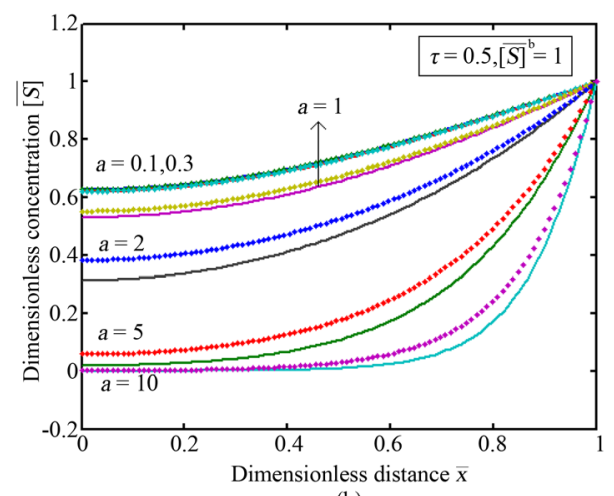

(b)

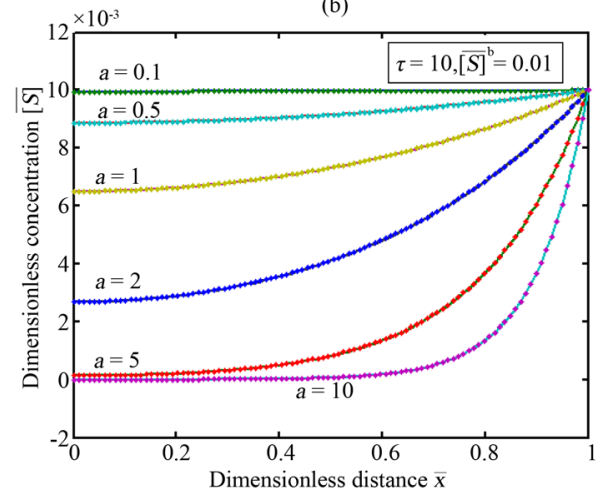

(d)

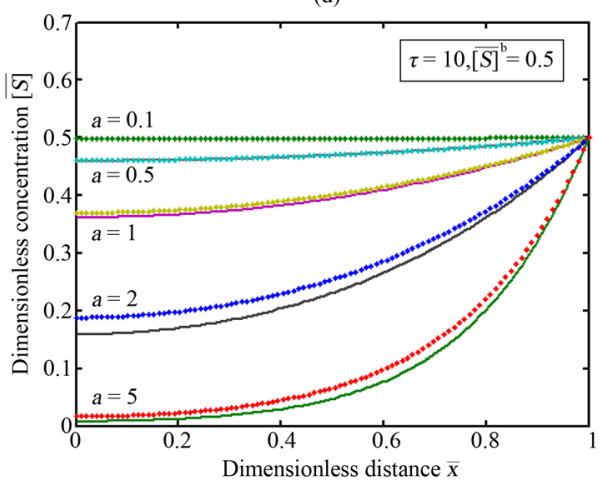

(f)

Figure 4. (a)-(f) Plot of dimensionless non-steady state concentration profiles of the substrate $\overline{[S}]$ versus dimensionless distance $\bar{x}$ for fixed values of time $\tau$ and $\overline{[S}^{b}$ and various values of the parameters $a$. Solid lines reprsent the Equation (15) and the dotted lines represent the numerical simulation. 
3) Influence of added buffer concentration on the concentration of species. The influence of added buffer concentration on the concentration of the substrate for some values of other parameters is shown in Figures 5(a)-(e). From this figure, it is inferred that the concentration of the substrate increases when added buffer concentration increases. Solid lines represent the Equation (15) and the dotted lines represent the numerical simulation. Satisfactory agreement is noted. The MATLAB program also given in Appendix B.

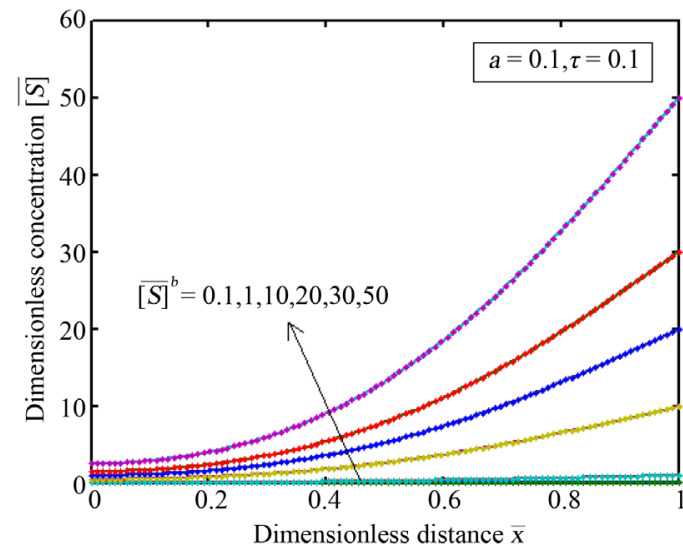

(a)

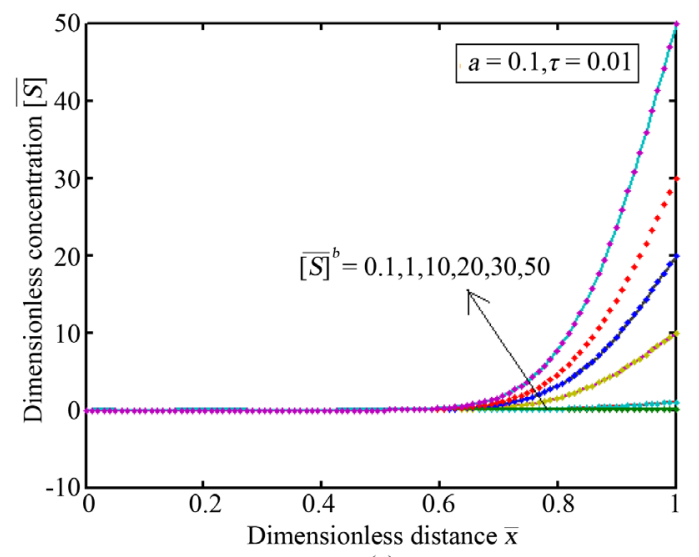

(c)

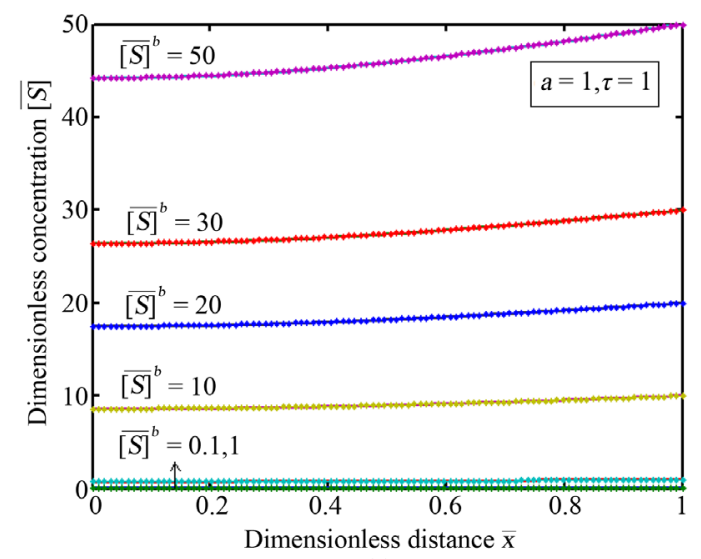

(e)

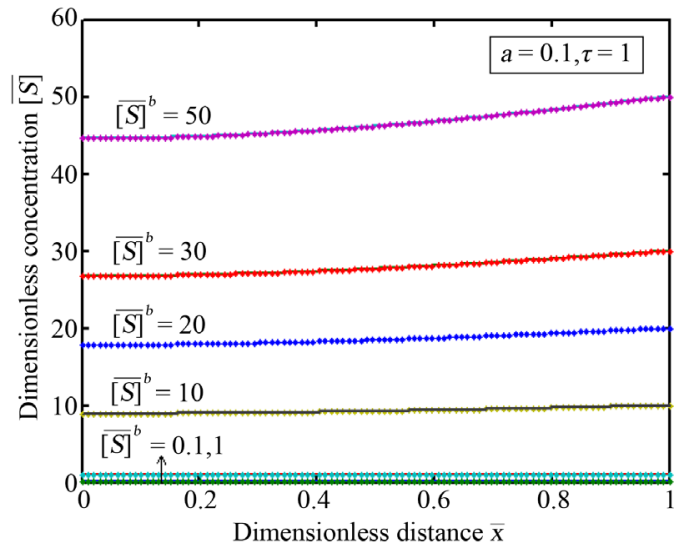

(b)

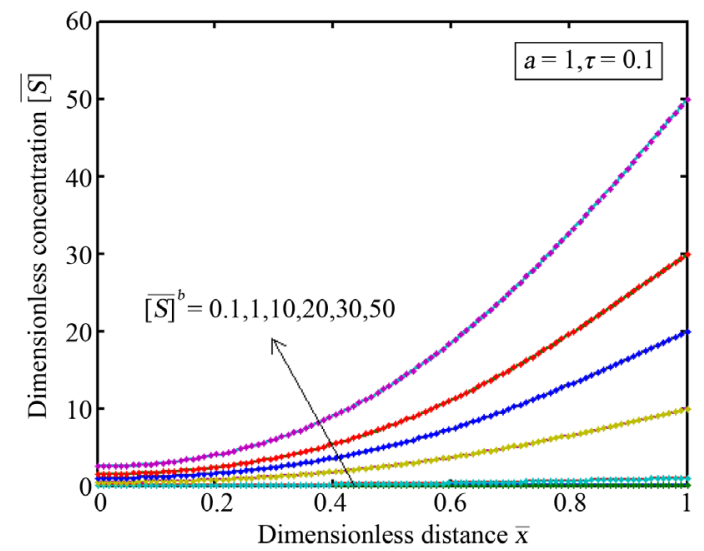

(d)

Figure 5. (a)-(e) Plot of dimensionless non-steady state concentration profiles of the substrate $\overline{[S]}$ versus dimensionless distance $\bar{x}$ for fixed values of $a$ and time $\tau$ and various values of the parameters $\overline{[S}^{b}$. Solid lines represent the Equation (15) and the dotted lines represent the numerical simulation. 
Figures 6(a)-(h) show the dimensionless non-steady state concentration profiles of products $\overline{\left[H^{+}\right]}, \overline{\left[P_{h}\right]_{T}}$ and $\overline{[Z]_{T}}$ versus dimensionless distance $\bar{x}$ for fixed values of $\overline{[S]^{b}}$ and $\tau$ and various values of the parameter $a$ using Equations (16), (17) and (18). From this Figure 6(a) and Figure 6(b), it is observed that the concentration of the product $\left[\mathrm{H}^{+}\right]$increases when Thiele modulus increases. Similarly, Figures 6(c)-(h) show that the concentration of the products $\overline{\left[P_{h}\right]_{T}}$ and $\overline{[Z]_{T}}$ increases when Thiele modulus increases.

Figure 7 (a) \& Figure 7 (b) show the dimensionless non-steady state concentration of substrate $\overline{[S]}$ and products $\left[\mathrm{H}^{+}\right]_{T},\left[\mathrm{P}_{h}\right]_{T}$ and $[\mathrm{Z}]_{T}$ versus dimensionless distance for various values of time $\tau$, using Equations (15)-(18).

Table 2 and Table 3 represents the comparison of analytical expression of concentration of the substrate $\bar{S}]$ (Equation (15)) with the numerical result for various values of parameter. In Table 2, average percentage of
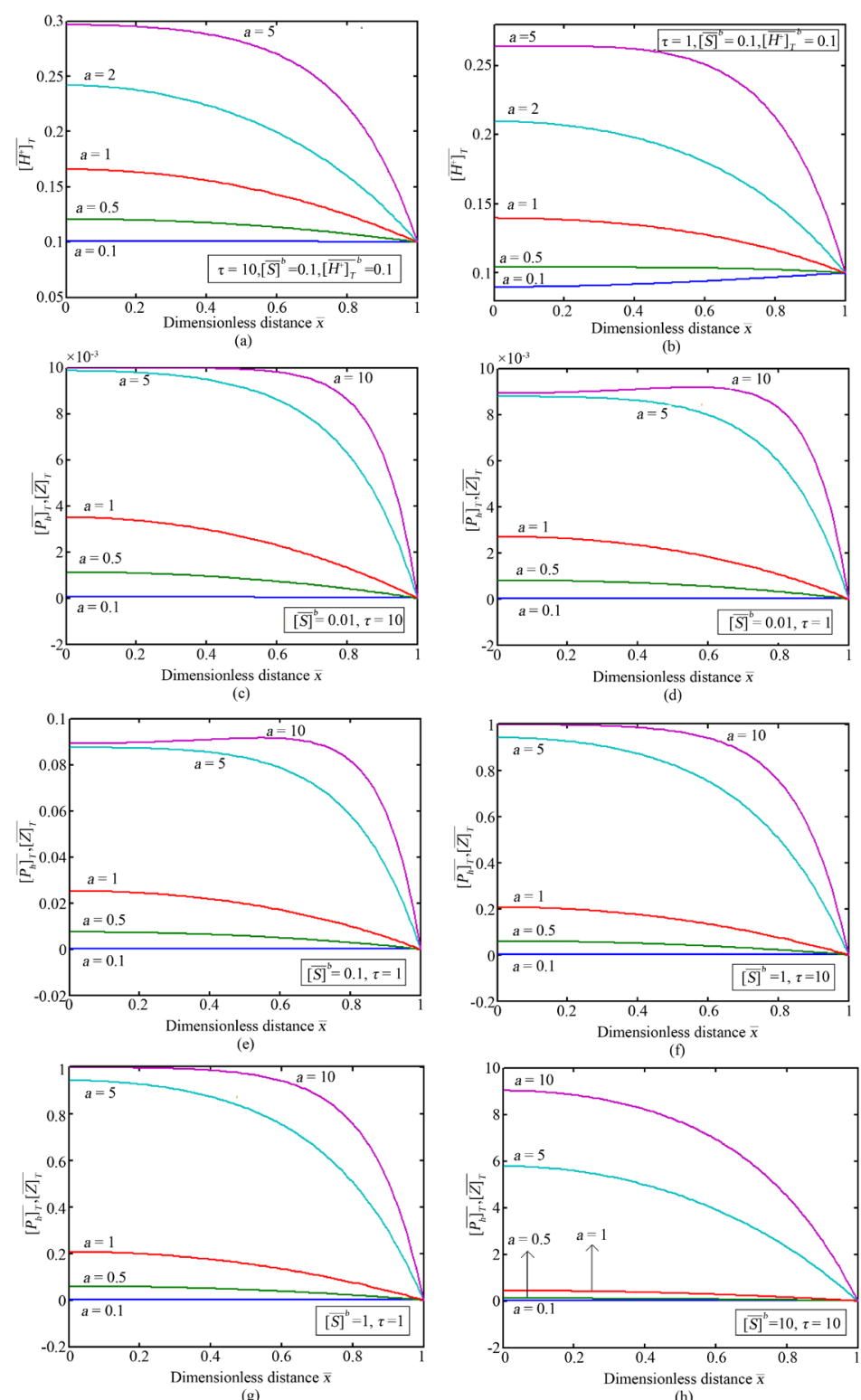

Figure 6. (a)-(h) Plot of dimensionless non-steady state concentration profiles of products $\overline{[Z]_{T}}, \overline{\left[H^{+}\right]_{T}}$ and $\overline{\left[P_{h}\right]_{T}}$ versus dimensionless distance $\bar{x}$ for fixed values of $\overline{[S}^{b}$ and $\tau$ and various values of the parameter $a$ using Equations (16), (17) and (18). 


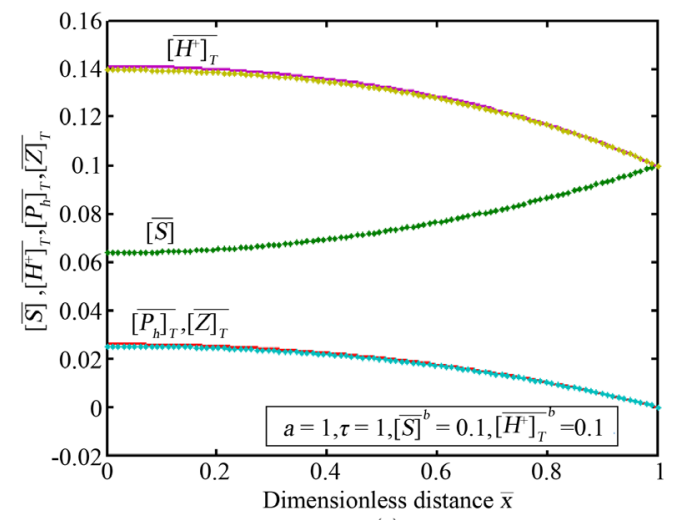

(a)

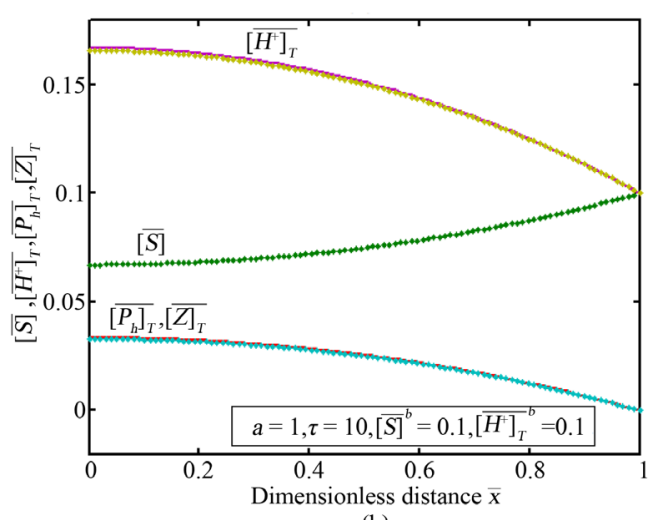

(b)

Figure 7. (a)-(b) Plot of dimensionless non-steady state concentration profiles of substrate $\overline{[S]}$ and products $\overline{\left[H^{+}\right]_{T}}$, $\overline{\left[P_{h}\right]_{T}}$ and $\overline{[Z]_{T}}$ versus dimensionless distance $\bar{x}$ for various values of time $\tau$, using Equations (15)-(18).

Table 2. Comparison of analytical expression of concentration of the substrate $\overline{[S]}$ (Equation (15)) with the numerical result for various values of parameter $a$.

\begin{tabular}{|c|c|c|c|c|c|c|c|c|c|}
\hline \multicolumn{4}{|c|}{$\overline{[S}^{b}=0.1, \tau=10, a=0.1$} & \multicolumn{3}{|c|}{$\overline{[S}^{b}=0.1, \tau=10, a=0.5$} & \multicolumn{3}{|c|}{$\overline{[S}^{b}=0.1, \tau=10, a=1$} \\
\hline $\bar{x}$ & Analytical & Numerical & $\%$ of deviation & Analytical & Numerical & $\%$ of deviation & Analytical & Numerical & $\%$ of deviation \\
\hline $\mathbf{0}$ & 0.09955 & 0.09955 & 0 & 0.08955 & 0.08962 & 0.07817 & 0.06650 & 0.06711 & 0.91729 \\
\hline 0.2 & 0.09955 & 0.09955 & 0 & 0.08996 & 0.09003 & 0.07781 & 0.06775 & 0.06834 & 0.87085 \\
\hline 0.4 & 0.09962 & 0.09962 & 0 & 0.09119 & 0.09126 & 0.07676 & 0.07155 & 0.07205 & 0.69881 \\
\hline 0.6 & 0.09971 & 0.09971 & 0 & 0.09327 & 0.09331 & 0.04289 & 0.07802 & 0.07840 & 0.48705 \\
\hline 0.8 & 0.09984 & 0.09984 & 0 & 0.09619 & 0.09622 & 0.03119 & 0.08740 & 0.08760 & 0.22883 \\
\hline 1 & 0.10000 & 0.10000 & 0 & 0.10000 & 0.10000 & 0 & 0.10000 & 0.10000 & 0 \\
\hline & \multicolumn{2}{|c|}{ Average $\%$ of deviation } & 0 & \multicolumn{2}{|c|}{ Average $\%$ of deviation } & 0.05114 & \multicolumn{2}{|c|}{ Average $\%$ of deviation } & 0.53381 \\
\hline
\end{tabular}

Table 3. Comparison of analytical expression of concentration of the substrate $\overline{[S]}$ (Equation (15)) with the numerical result for various values of parameter $\tau$.

\begin{tabular}{|c|c|c|c|c|c|c|c|c|c|c|c|c|}
\hline & \multicolumn{3}{|c|}{$\tau=0.1, \overline{[S]}^{b}=0.1, a=0.1$} & \multicolumn{3}{|c|}{$\tau=0.5, \overline{[S}^{b}=0.1, a=0.1$} & \multicolumn{3}{|c|}{$\tau=1, \overline{[S}]^{b}=0.1, a=0.1$} & \multicolumn{3}{|c|}{$\tau=10, \overline{[S}^{b}=0.1, a=0.1$} \\
\hline $\bar{x}$ & Analytical & Numerical & $\begin{array}{c}\% \text { of } \\
\text { deviation }\end{array}$ & Analytical & Numerical & $\begin{array}{c}\% \text { of } \\
\text { deviation }\end{array}$ & Analytical & Numerical & $\begin{array}{c}\text { \% of } \\
\text { deviation }\end{array}$ & Analytical & Numerical & $\begin{array}{c}\% \text { of } \\
\text { deviation }\end{array}$ \\
\hline $\mathbf{0}$ & 0.00507 & 0.00500 & 1.38067 & 0.06276 & 0.06271 & 0.07967 & 0.08886 & 0.08885 & 0.01125 & 0.09955 & 0.09955 & 0 \\
\hline 0.2 & 0.00809 & 0.00802 & 0.86527 & 0.06458 & 0.06453 & 0.07742 & 0.08940 & 0.08940 & 0 & 0.09957 & 0.09957 & 0 \\
\hline 0.4 & 0.01814 & 0.01806 & 0.44101 & 0.06986 & 0.06981 & 0.07157 & 0.09097 & 0.09097 & 0 & 0.09962 & 0.09962 & 0 \\
\hline 0.6 & 0.03713 & 0.03704 & 0.24239 & 0.07809 & 0.07804 & 0.06403 & 0.09343 & 0.09342 & 0.0107 & 0.09971 & 0.09971 & 0 \\
\hline 0.8 & 0.06546 & 0.06536 & 0.15277 & 0.08847 & 0.08843 & 0.04521 & 0.09653 & 0.09653 & 0 & 0.09984 & 0.09984 & 0 \\
\hline \multirow[t]{2}{*}{1} & 0.10000 & 0.09991 & 0.09 & 0.10000 & 0.09997 & 0.03 & 0.10000 & 0.09999 & 0.01 & 0.10000 & 0.10000 & 0 \\
\hline & \multicolumn{2}{|c|}{$\begin{array}{c}\text { Average \% of } \\
\text { deviation }\end{array}$} & 0.52869 & \multicolumn{2}{|c|}{$\begin{array}{c}\text { Average \% of } \\
\text { deviation }\end{array}$} & 0.06132 & \multicolumn{2}{|c|}{$\begin{array}{c}\text { Average \% of } \\
\text { deviation }\end{array}$} & 0.00533 & \multicolumn{2}{|c|}{$\begin{array}{c}\text { Average \% of } \\
\text { deviation }\end{array}$} & 0 \\
\hline
\end{tabular}


error deviation increases, when the reaction diffusion parameter $a$ increases. Similarly in Table 3, average percentage of error deviation decreases, when the time $\tau$ increases. In Table 2, the maximum average relative error between the analytical results and numerical results is $0.53 \%$.

\section{Conclusion}

A non-linear time dependent system of differential equation in pH-based potentiometric biosensor has been solved using the new HPM. New approximate analytical expressions for the concentrations of the substrate and hydrolysis products are derived. The time dependent substrate concentration profiles are also presented using SCILAB program. Concentration of substrate and product depends upon Thiele modulus and initial concentration of substrate which is discussed in this communication.

\section{Acknowledgements}

This work was supported by the DST SB/SI/PC-50/2012, New Delhi, India. The authors are thankful to Mr. S. Mohamed Jaleel, The Chairman, Dr. A. Senthilkumar, The Principal, Dr. P. G. Jansi Rani, Head of the Department of Mathematics, Sethu Inistitute of Technology, Kariapatti-626115, Tamilnadu, India for their encouragement.

\section{References}

[1] Baronas, R., et al. (2010) Mathematical Modeling of Biosensors: An Introduction for Chemists and Mathematicians. Springer. http://dx.doi.org/10.1007/978-90-481-3243-0

[2] Sahin, A. (2012) Development of Electrochemical Methods for Detection of Pesticides and Biofuel Production. Ph.D. Thesis, Columbia University Academic Commons.

[3] Rajendran, L. (2013) Chemical Sensors Simulation and Modeling, Electrochemical Sensors. In: Korotchenkov, G., Ed.

[4] Meena, A. and Rajendran, L. (2010) Analysis of a pH-Based Potentiometric Biosensor Using the Homotopy Perturbation Method. Chemical Engineering \& Technology, 33, 1-10. http://dx.doi.org/10.1002/ceat.200900580

[5] Rahamathunissa, G. and Rajendran, L. (2008) Application of He’s Variational Iteration Method in Nonlinear Boundary Value Problems in Enzyme-Substrate Reaction Diffusion Processes: Part 1. The Steady-State Amperometric Response. Journal of Mathematical Chemistry, 44, 849-861. http://dx.doi.org/10.1007/s10910-007-9340-9

[6] Manimozhi, P., Subbiah, A. and Rajendran, L. (2010) Solution of Steady-State Substrate Concentration in the Action of Biosensor Response at Mixed Enzyme Kinetics. Sensors and Actuators B, 147, 290-297. http://dx.doi.org/10.1016/j.snb.2010.03.008

[7] Dong, S. and Che, G. (1991) Electrocatalysis at a Microdisk Electrode Modified with a Redox Species. Journal of Electroanalytical Chemistry, 309, 103-114. http://dx.doi.org/10.1016/0022-0728(91)87007-Q

[8] Lyons, M.E.G., Bannon, T. and Rebouillat, S. (1998) Reaction/Diffusion at Conducting Polymer Ultramicroelectrodes. Analyst, 123, 1961-1966. http://dx.doi.org/10.1039/a804039g

[9] Eswari, A. and Rajendran, L. (2010) Analytical Solution of Steady-State Current an Enzyme-Modified Microcylinder Electrodes. Journal of Electroanalytical Chemistry, 648, 36-46. http://dx.doi.org/10.1016/j.jelechem.2010.07.002

[10] Wang, L.H., Zhang, L., Huang, J.X. and Chen, H.L. (2006) Model of a pH-Based Potentiometric Biosensor Immobilizing Organophosphorus Hydrolase. Chemical Engineering \& Technology, 29, 462-467. http://dx.doi.org/10.1002/ceat.200500281

[11] He, J.-H. (2006) Some Asymptotic Methods for Strongly Nonlinear Equations. International Journal of Modern Physics, 20, 1141-1199. http://dx.doi.org/10.1142/S0217979206033796

[12] He, J.-H. (1999) Homotopy Perturbation Technique. Computer Methods in Applied Mechanics and Engineering, 178, 257-262. http://dx.doi.org/10.1016/S0045-7825(99)00018-3

[13] Rajendran, L. and Anitha, S. (2013) Comments on Analytical Solution of Amperometric Enzymatic Reactions Based on HPM. Electrochimica Acta, 102, 474-476. http://dx.doi.org/10.1016/j.electacta.2013.03.163 


\section{Appendix A. The Dimensionless Reaction-Diffusion Equations}

In the enzyme membrane, the reaction-diffusion equations for the concentration of species for non-steady state condition can be represented as follows [4] [10].

$$
\begin{gathered}
\frac{\partial[S]}{\partial t}=D \frac{\partial^{2}[S]}{\partial x^{2}}-R \\
\frac{\partial\left[P_{h} H\right]}{\partial t}=D \frac{\partial^{2}\left[P_{h} H\right]}{\partial x^{2}}+R-r_{P_{h} H} \\
\frac{\partial\left[P_{h}^{-}\right]}{\partial t}=D \frac{\partial^{2}\left[P_{h}^{-}\right]}{\partial x^{2}}+r_{P_{h} H} \\
\frac{\partial[Z H]}{\partial t}=D \frac{\partial^{2}[Z H]}{\partial x^{2}}+R-r_{Z H} \\
\frac{\partial\left[Z^{-}\right]}{\partial t}=D \frac{\partial^{2}\left[Z^{-}\right]}{\partial x^{2}}+r_{Z H} \\
\frac{\partial[A H]}{\partial t}=D \frac{\partial^{2}[A H]}{\partial x^{2}}-r_{A H} \\
\frac{\partial\left[A^{-}\right]}{\partial t}=D \frac{\partial^{2}\left[A^{-}\right]}{\partial x^{2}}+r_{A H} \\
\frac{\partial\left[H^{+}\right]}{\partial t}=D \frac{\partial^{2}\left[H^{+}\right]}{\partial x^{2}}+r_{P_{h} H}+r_{Z H}+r_{A H}
\end{gathered}
$$

where $r_{P_{h} H}, r_{Z H}, r_{A H}, r_{A H}$ are the instantaneous reaction terms. We also assume that the substrate $S$ reacts with the catalysts via Michaelis-Menten kinetics. The reaction rate is

$$
R=\frac{v_{\max }[S]}{[S]+k_{m}}
$$

where

$$
v_{\max }=\frac{k_{2} k_{3}[E]_{t}}{\left(k_{2}+k_{3}\right)}, k_{m}=\frac{\left(k_{2}+k_{-1}\right)}{\left(k_{2}+k_{3}\right)} \frac{k_{3}}{k_{1}}
$$

By introducing the following set of dimensionless variables

$$
\overline{C_{i}}=\frac{C_{i}}{k_{m}} ; \bar{x}=\frac{x}{L} ; a^{2}=\frac{L^{2} V_{\max }}{D k_{m}} ; \tau=\frac{D t}{L^{2}}
$$

and defining the following "composite species" [10]

$$
\begin{gathered}
{\left[P_{h}\right]_{T}=\left[P_{h} H\right]+\left[P_{h}^{-}\right]} \\
{[Z]_{T}=[Z H]+\left[\mathrm{Z}^{-}\right]} \\
{[A]_{T}=[\mathrm{AH}]+\left[\mathrm{A}^{-}\right]} \\
{\left[\mathrm{H}^{+}\right]_{T}=\left[\mathrm{H}^{+}\right]+\left[\mathrm{P}_{h} H\right]+[\mathrm{ZH}]+[\mathrm{AH}]}
\end{gathered}
$$

we obtain the dimensionless form of Equations (6)-(10) for the concentration of species which are given in the text. 


\section{Appendix B. Analytical Solutions of Equations (6)-(10) Using Complex Inversion} Formula

In this appendix, we indicate how the Equations (15) is derived. Using new homotopy perturbation approach [13], Equation (6) can be written as

$$
\begin{aligned}
& (1-p)\left[\frac{\partial^{2} \overline{[S]}}{\partial \bar{x}^{2}}-a^{2} \frac{\overline{[S]}}{\overline{[S]}[\bar{x}=1]+1}-\frac{\partial \overline{[S]}}{\partial \tau}\right]+p\left[\frac{\partial^{2} \overline{[S]}}{\partial \bar{x}^{2}}-a^{2} \frac{\overline{[S]}}{\overline{[S]}+1}-\frac{\partial \overline{[S]}}{\partial \tau}\right]=0 \\
& (1-p)\left[\frac{\partial^{2} \overline{[S]}}{\partial \bar{x}^{2}}-a^{2} \frac{\overline{[S]}}{\overline{[S]^{b}+1}}-\frac{\partial \overline{[S]}}{\partial \tau}\right]+p\left[\frac{\partial^{2} \overline{[S]}}{\partial \bar{x}^{2}}-a^{2} \frac{\overline{[S]}}{\overline{[S]}+1}-\frac{\partial \overline{[S]}}{\partial \tau}\right]=0
\end{aligned}
$$

The approximate solution of Equation (B.2) is

$$
\overline{[S]}=\overline{[S]_{0}}+p \overline{[S]_{1}}+p^{2} \overline{[S]_{2}}+\cdots
$$

Substituting Equation (B.3) into Equation (B.2) and arranging the coefficients of powers $p$

$$
\begin{gathered}
p^{0}: \frac{\partial^{2} \overline{[S]_{0}}}{\partial \bar{x}^{2}}-a^{2} \frac{\overline{[S]_{0}}}{\overline{[S]^{b}}+1}-\frac{\partial \overline{[S]_{0}}}{\partial \tau}=0 \\
p^{1}: \frac{\partial^{2} \overline{[S]_{1}}}{\partial \bar{x}^{2}}-a^{2} \frac{\overline{[S]_{1}}}{\overline{[S]^{b}}+1}-\frac{\partial \overline{[S]_{1}}}{\partial \tau}+a^{2} \frac{\overline{[S]_{0}}}{\overline{[S]^{b}+1}}-a^{2} \frac{\overline{[S]_{0}}}{\overline{[S]_{0}}+1}=0
\end{gathered}
$$

The initial and boundary conditions for Equations (12)-(14) becomes

$$
\begin{aligned}
& \text { At } \tau=0, \overline{[S]_{0}}=0 \text { and } \overline{[S]_{i}}=0 \quad \forall i=1,2,3, \cdots \\
& \text { At } \bar{x}=0, \frac{\mathrm{d} \overline{\overline{[S]_{0}}}}{\mathrm{~d} \bar{x}}=0 \text { and } \forall i=1,2,3, \cdots \\
& \text { At } \bar{x}=1, \overline{[S]_{0}}=\overline{[S]^{b}} \text { and } \overline{[S]_{i}}=0 \forall i=1,2,3, \cdots
\end{aligned}
$$

Equation (B.4) can be written as

$$
\frac{\partial \overline{[S]_{0}}}{\partial \tau}=\frac{\partial^{2} \overline{[S]_{0}}}{\partial \bar{x}^{2}}-\alpha \overline{[S]_{0}}
$$

where $\alpha$ is defined as in Equation (20). Now, by applying Laplace transform and complex inversion formula (Appendix C) to Equation (B.9) and to the conditions in Equations (B.6)-(B.8), we obtained the solution of Equation (B.9) as

$$
\overline{\overline{[S]_{0}}}=\frac{\overline{[S]^{b}} \cosh (\sqrt{s+\alpha} \bar{x})}{s \cosh (\sqrt{s+\alpha})}
$$

Using residue theorem (Appendix C) we can obtain the Equation (15) in the text.

\section{Appendix C. Inverse of Equation (B. 10) by Using Complex Inversion Formula}

In this appendix, we indicate how Equation (B.10) may be inverted using the complex inversion formula. If $\bar{y}(s)$ represents the Laplace transform of a function $\bar{y}(\tau)$, then, according to the complex inversion formula, we can state that

$$
\bar{y}(\tau)=\frac{1}{2 \pi \int_{c-i \infty}^{c+i \infty} \exp [s \tau] \bar{y}(s) \mathrm{d} s}=\frac{1}{2 \pi i} \oint_{c} \exp [s \tau] \bar{y}(s) \mathrm{d} s
$$


where the integration in Equation (C.1) is to be performed along a line $s=c$ in the complex plane where $s=x+i y$. The real number $c$ is chosen such that $s=c$ lies to the right of all the singularities but is otherwise assumed to be arbitrary. In practice, the integral is evaluated by considering the contour integral presented on the right-hand side of Equation (C.1), which is then evaluated using the so-called Bromwich contour. The contour integral is then evaluated using the residue theorem which states, for any analytic function $F(z)$, that

$$
\oint_{C} F(z) \mathrm{d} z=2 \pi i \sum_{n} \operatorname{Res}[F(z)]_{z=z_{0}}
$$

where the residues are computed at the poles of the function $F(z)$. Hence, from Equation (C.2), we note that

$$
y(\tau)=\sum_{n} \operatorname{Res}[\exp [s \tau] \bar{y}(s)]_{s=s_{0}}
$$

From the theory of complex variables, we can show that the residue of a function $F(z)$ at a simple pole at $\mathrm{Z}=a$ is given by

$$
\operatorname{Res}[F(z)]_{z=a}=\lim \{(z-a) F(z)\}
$$

Hence, in order to invert Equation (B.10), we need to evaluate

$$
\operatorname{Res}\left[\frac{\overline{[S]^{b}} \cosh (\sqrt{s+\alpha} \bar{x})}{s \cosh (\sqrt{s+\alpha})}\right]
$$

The poles are obtained from $s \cosh (\sqrt{s+\alpha})=0$. Hence, there is a simple pole at $s=0$ and there are infinitely many poles given by the solution of the equation $\cosh (\sqrt{s+\alpha})=0$ and so

$$
s_{n}=\frac{-\pi^{2}(2 n+1)^{2}-4 \alpha}{4} \text { where } n=0,1,2, \cdots
$$

Hence, we note that

$$
\overline{[S]_{0}}(\bar{x}, \tau)=\overline{[S}^{b}\left\{\operatorname{Res}[s \cosh (\sqrt{s+\alpha})]_{s=0}+\operatorname{Res}[s \cosh (\sqrt{s+\alpha})]_{s=s_{n}}\right\}
$$

The first residue in Equation (B.17) is given by

$$
\operatorname{Res}[s \cosh (\sqrt{s+\alpha})]_{s=0}=\lim \left[\frac{\exp (s \tau) \cosh (\sqrt{s+\alpha}) \bar{x}}{s \cosh (\sqrt{s+\alpha})}\right]=\frac{\cosh \sqrt{\alpha} \bar{x}}{\cosh \sqrt{\alpha}}
$$

The second residue in Equation (B.17) is given by

$$
\begin{aligned}
& \operatorname{Res}[s \cosh (\sqrt{s+\alpha})]_{s=s_{n}} \\
& =\lim \left[\frac{\exp (s \tau) \cosh (\sqrt{s+\alpha}) \bar{x}}{s \cosh (\sqrt{s+\alpha})}\right]=\lim \left[\frac{\exp (s \tau) \cosh (\sqrt{s+\alpha}) \bar{x}}{s(\mathrm{~d} / \mathrm{d} s) \cosh (\sqrt{s+\alpha})}\right] \\
& =-\sum_{n=0}^{\infty}\left[\frac{(-1)^{n} \pi(2 n+1) \mathrm{e}^{-f_{n} \tau} \cos ((2 n+1) \pi \bar{x} / 2)}{f_{n}}\right]
\end{aligned}
$$

where $f_{n}$ is defined as in Equation (20). Here, we used $\cosh (i \theta)=\cos (\theta)$ and $\sinh (i \theta)=i \sin (\theta)$. From Equations (C.7)-(C.9), we conclude that

$$
\overline{[S]_{0}}(\bar{x}, \tau)=\overline{[S]}\left\{\frac{\cosh \sqrt{\alpha} \bar{x}}{\cosh \sqrt{\alpha}}-\sum_{n=0}^{\infty}\left[\frac{(-1)^{n} \pi(2 n+1) \mathrm{e}^{-f_{n} \tau} \cos ((2 n+1) \pi \bar{x} / 2)}{f_{n}}\right]\right\}
$$

where $f_{n}$ is defined as in Equation(20). Similarly, we can solve Equations (7) to (10) by using complex inversion formula. 
Appendix D. Scilab/Matlab Program to Find the Numerical Solutions of Equations (15) to (18)

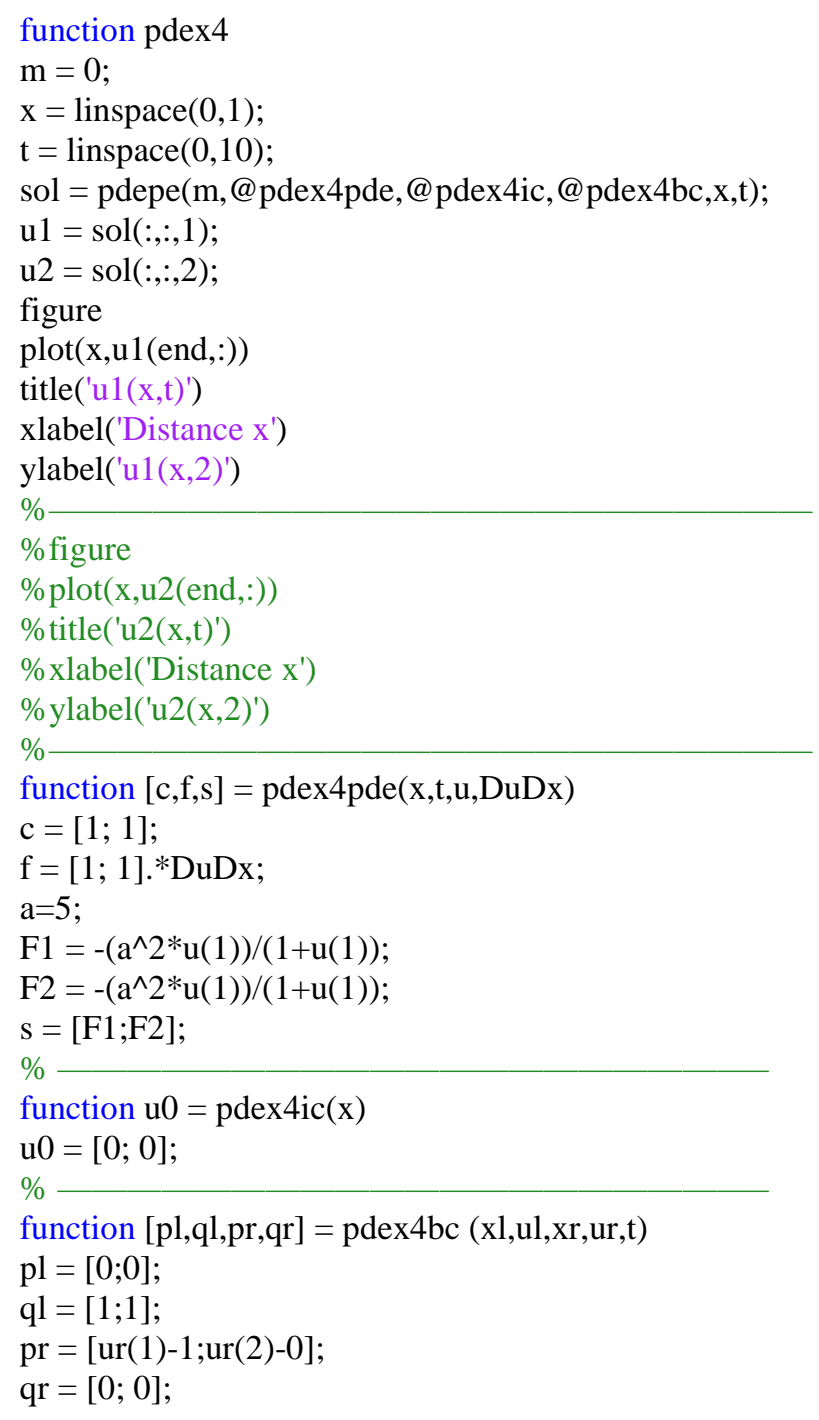

